\title{
Research Paper: Attentional Bias and Prediction of Attempted Suicide in Clinical and Non-clinical Population
}

\author{
Elham Baghani $^{1}$ (D), Ladan Fata ${ }^{{ }^{*}}$ (D, Mahdiyeh Salehi ${ }^{1}$ (D) Fariba Hasani $^{1}$ (D)
}

1. Department of General Psychology, Central Tehran Branch, Islamic Azad University, Tehran, Iran.

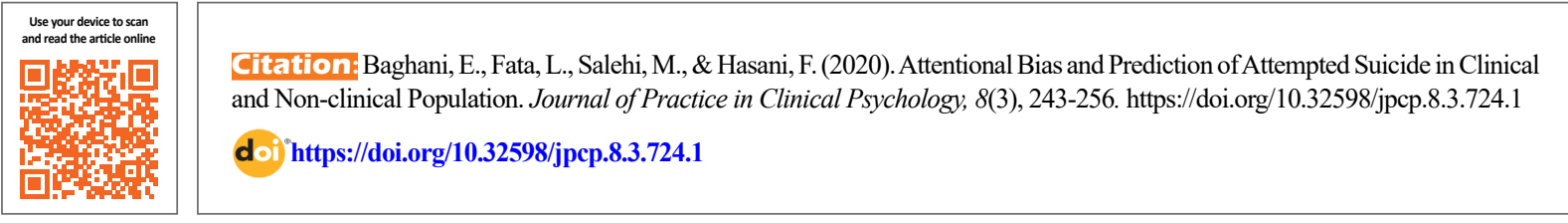

\section{(C) $(1) \Theta$}

Article info:

Received: 16 Sep 2019

Accepted: 15 Jun 2020

Available Online: $01 \mathrm{Jul} 2020$

\section{Keywords:}

Stroop test, Attentional bias, Attempted suicide

\section{A B STRACT}

Objective: the aim of the present research was to analyze the predictability of suicide attempts, based on attentional bias in a clinical and non-clinical population.

Methods: 120 participants (77 females and 43 males) were intentionally selected in three diagnostic groups: clinical suicides, clinical non-suicides, and non-clinical ones (40 individuals in each group). They participated in the Suicide Stroop Task as well as the Beck Scale of Suicide Ideation (BSS). The attentional bias parameters, including reaction time and interference time, were computed and the data were analyzed using Multivariate Analysis of Variance and Discriminant Analysis.

Results: the results obtained from the multivariate analysis revealed that the variables of interference time did not have a significant indication for the three groups of individuals in suicide stroop ( $\mathrm{P}<0.568 ; \mathrm{F}=0.569)$, positive stroop $(\mathrm{P}<0.201 ; \mathrm{F}=1.626)$, and negative stroop $(\mathrm{P}<0.863$; $\mathrm{F}=0.147)$. However, in the case of reaction time, there was a significant difference in the values obtained for all the three groups in terms of suicide stroop $(\mathrm{P}<0.000 ; \mathrm{F}=12.759)$, positive stroop $(\mathrm{P}<0.000 ; \mathrm{F}=18.520)$, negative stroop $(\mathrm{P}<0.000 ; \mathrm{F}=10.995)$, and neutral stroop $(\mathrm{P}<0.000$; $\mathrm{F}=8.288$ ). In addition, the results obtained from the discriminant analysis show that the variables of suicide ideation (0.822), reaction time in positive stroop (0.571), suicide stroop (0.470), negative stroop (0.438), and neutral stroop (0.376) were correspondingly crucial in predicting the behavior of the clinical suicide attempt, clinical non-suicide attempt, and non-clinical groups.

Conclusion: We found that for suicide stroop, the clinical and non-clinical cases did not show a significant difference in terms of the interference effect; however, they differed in terms of reaction time. Therefore, it seems that using emotional stroop, attentional bias towards suiciderelated topics is not dependent on the clinical situations, and other parameters, such as scoring methods should be considered. However, there is still a need to conduct more extensive studies.

* Corresponding Author:

Ladan Fata, PhD.

Address: Department of General Psychology, Central Tehran Branch, Islamic Azad University, Tehran, Iran.

Tel: +98 (21) 22481632

E-mail: lfata@yahoo.com 


\section{Highlights}

- Attentional bias, which includes selective allocation of attention resources to some specific aspects of the stimulus, is a cognitive process that helps more to explain and predict suicidal behaviors. Suicide Stroop Test is a modified version of the emotional Stroop test that measures the degree of the interference created by strict processing to support pioneer responses to a stimulus with a given emotion (Cisler, Bacon, \& Williams, 2009).

- According to the results obtained from the analyses, the interactive effect of the group, and the Stroop type on the attentional bias is not significant based on none of the scoring methods for the two categories. However, based on the reaction time scoring method, the group's effect is substantial on attentional bias in both dual and triple categories.

- The results indicated that attentional bias analysis, based on the interference effect, does not provide a significant difference between the three groups. Thus it cannot play any significant role in predicting the groups. However, when reaction time is used as the criterion, all three groups of emotional words (suicide, positive and negative) produce a significant difference between the groups.

- It seems that the assessment of attentional bias, using emotional Stroop with respect to suicide-related topics, is not a function of the clinical situation, and other factors, such as the scoring method, are involved. So further extensive investigations should be conducted in this area.

\section{Plain Language Summary}

The present research, as the first study in Iran, uses suicide Stroop to predict suicidal behaviors. It aims to analyze the predictability of suicide attempts based on attentional bias in a clinical population (suicidal and non-suicidal) and nonclinical one. The main objective of the present research was to assess and compare the predictive role of attentional bias in suicidal clinical, suicidal non-clinical, and non-clinical patients. Attentional bias, which includes selective allocation of attention resources to some specific aspects of the stimulus, is a cognitive process that helps more to explain and predict suicidal behaviors. In a suicidal person, a semi-conscious and semi-attention bias used to record such stimuli according to the schemas' content might make the stimuli conquer the person's attention. Thus, the information related to the stimuli might be processed and selectively encoded in the memory, and this is when the reliable data will be ignored. The results indicated that attentional bias analysis, based on the interference effect, does not provide a significant difference between the three groups. Thus it cannot play any significant role in predicting the groups. However, when reaction time is used as the criterion, all three groups of emotional words (suicide, positive and negative) produce a significant difference between the groups. In the present research, clinical and non-clinical populations studied in suicide Stroop with respect to interference did have a considerable difference. However, they were different in terms of reaction time. Hence, it seems that the assessment of attentional bias, using emotional Stroop with respect to suicide-related topics, is not a function of the clinical situation, and other factors, such as the scoring method, are involved. So, further extensive investigations should be conducted in this area.

\section{Introduction}

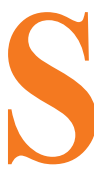

uicide is one of the most important issues of general health (Wenzel, Brown $\&$ Beck, 2009). Recent statistics, reported by the World Health Organization (WHO) in 2019, show that approximately 800,000 individuals die because of suicide every year, which corresponds to one person for every $40 \mathrm{~s}$. According to the WHO's estimates, based on the current trends, it is expected that the number of people with completed suicide will increase to 1.5 mil- lion in 2020 (WHO, 2019). However, the number of people who attempt suicide will be 10-20 times more than this rate. Although these statistics are approximate, they indicate an important concern that should be considered, otherwise the world will face a disaster. In the case of Iran, an accurate and comprehensive statistical system has not yet been developed to report the suicide rate and the statistics are only determined based on the reports, provided by the Iranian Legal Medicine Organization. The recent report by this organization published in 2018-2019 shows that the rate of a suicide attempt is 125.24 persons for every 100,000 individuals, which 
indicates a 5\% increase in the rate compared with last year's report (Young Journalists Club, 2018).

Different factors can distinguish people who attempt suicide from those that do not attempt. In recent decades, predicting suicide ideations and behaviors, as well as the methods used to evaluate suicidal risk, has become an area of interest in health research, which challenges investigators. Several indirect methods have been introduced, which were used to predict suicidal behaviors and evaluate suicidal risk. These methods include the Kesler's 10-item syndrome checklist (k-10), formal analysis of the suicidal situation (Hamedi, Colborn, Bell, Chalker, \& Jobes, 2019), Eye-Blink/Startle Response Paradigm (Goodman \& Hazlett, 2015), thermography technique to record auto-physiological responses, such as the opening of sweat pores while answering to the questions regarding the suicide (Familoni et al., 2012), Implicit Association Test (Nock et al., 2010) and Suicide Stroop Task (SST) (Chung \& Jeglic, 2016; Richard-Devantoy, Ding, Turecki \& Jollant, 2016; Wilson et al., 2019; Becker, Strohbach \& Rinck, 1999; Williams \& Broadbent, 1986; Cha, Najmi, Park, Finn \& Nock, 2010; Stewart et al., 2017). Some of these methods, such as the SST, focus on "Attentional Bias" in case of suicide information processing. Attentional bias, which includes selective allocation of attention resources to some specific aspects of the stimulus, is a cognitive process, which helps explain and predict suicidal behaviors.

Attentional bias or selective process of information (data) is rooted in a group of theoretical formulations and empirical experiments. There are some particular schemas in the long-term memory that has an active role in organizing the flow of new information. When a schema is activated and influences the selection of the next stimuli, supposed to be targeted, it facilitates the retrieval of relative topics from the memory and determines the general importance of the corresponding stimulus for an individual (Turner \& Beidel, 2004). In the case of a suicidal person, a semi-conscious and semi-attention bias, used to record such stimuli according to the content of the schemas, might conquer the person's attention by the stimuli; thus, the information, related to the stimuli, might be processed and encoded in the memory in a selective manner when the reliable information will be ignored.

The final result might be producing an active schema in memory that can also easily appear in vague situations (Davidson, 2000). According to the cognitive model for suicide attempts (Wenzel et al., 2009), individuals, who have attention bias and fixational attention, cannot have a general distinction in the procedure of information pro- cessing. These individuals are not able to make an appropriate judgment about their situation and consequently, they are fixed on the suicide option (Wenzel et al., 2009). SST is a modified version of the emotional stroop test that measures the degree of the interference created by strict processing to support pioneer responses to a stimulus with a given emotion (Cisler, Bacon \& Williams, 2009). In SST, the words are presented as stimuli of suicide-related topics, while it is performed as the cognitive task. The person is asked to recognize the color of the words without considering their meanings. In case of attention bias towards suicide-related words, the individual is not able to complete the cognitive task as soon as possible and this delay in responding to the task, which is the result of the cognitive interference, is deemed as the attention bias to those suicide-related stimuli (Wilson et al., 2019).

For instance, Williams \& Broadbent (1986) reported a difference in attention bias, which is defined based on a delay response in the Autobiographical Memory Retrieval Test, among three groups of people, including a clinical suicide attempt, clinical non-suicide attempt, and nonclinical people. Becker et al. (1999) revealed that a group of clinical patients, with a history of suicide, have a longer response in suicide stroop (i.e. neutral and emotional, positive or negative). Their results clearly expressed that for the clinical group, the attentional bias was associated with the suicide-related information, while in the non-clinical group, there was no difference in reaction time to different types of stroop. Cha et al. (2010) using a computational stroop test showed that those with a history of suicide suffer from attentional bias in suicide stroop. On the other hand, those who did not attempt suicide had negative bias (i.e. faster reaction time to suicide stroop than neutral stroop). In contrast to conventional hypotheses and conclusions regarding the predictive value of attention bias, other investigations present inconsistent results.

For instance, Chung \& Jeglic (2016) reported that in terms of a delay in negative emotional stroop and suicide stroop, there is no significant difference between people with a history of suicide in comparison with those who did not have such a history. In addition, according to Richard-Devantoy, Ding, Turecki \& Jollant (2016), comparing attentional bias with respect to negative and positive emotional stroop and suicide stroop, there is no significant difference in various types of stroop between clinical suicide attempt individuals and those with mood disorders. Despite the importance of attentional bias in various types of psychopathologies, there is still a big gap between the available knowledge and its practical applications. According to contemporary cognitive psychologists, the objective of new behavioral therapies is 
to change, restore, and repair cognitive processes. On the other hand, according to a high rate of suicidal behaviors, it is crucial to identify different factors involved in the occurrence of such behaviors and design effective and scientific-based interventions to prevent it at the population, group, and individual levels (WHO, 2019). For this purpose, the present research- as the first study in Iran, which uses suicide stroop to predict suicidal behaviorswas done to analyze the predictability of suicide attempt, based on attentional bias in clinical (suicidal and nonsuicidal) and non-clinical populations.

\section{Methods}

The present cross-sectional study was conducted on individuals, who attempted to suicide and psychiatric outpatients, referred to hospitals, clinics, and health centers in Tehran (particularly Loghman-e Hakim Hospital, to which self-poisoning patients with suicidal intention are usually referred) and other available non-clinical individuals, such as students, co-workers, and people with whom the authors are living and working. Accordingly, 120 individuals (40 cases for the suicidal clinical group, 40 cases for the non-suicidal clinical group, and 40 cases for the non-clinical group) consisting of 77 females $(62.4 \%)$ and 43 males $(35.8 \%)$ were selected. In terms of age, 44 cases $(36.7 \%)$ are younger than 20 years, 63 cases $(52.5 \%)$ were between 20 and 30 years, and $43(10.8 \%)$ cases were older than 30 years. Sampling was done purposely, based on the accessibility of the statistical population. For different groups, frequency and percentage of the demographic characteristics of the research sample are presented in Table 1.

Participants of the suicidal clinical group were exclusively those who attempted suicide and they were hospitalized. The non-suicidal clinical group was psychiatric outpatients who did not commit suicide since last year. The non-clinical group consisted of those selected based on the General Health Questionnaire (GHQ) and in terms of clinical indicators (Goldberg \& Hillier, 1979). The following inclusion criteria were considered: the age range of 18 to 40 years, being literate and having consent to attend to this study. In particular, the inclusion criterion for the suicidal clinical group was the accurate confirmation of committing suicide using any medicine or other poisons and being hospitalized. In addition, these cases needed to have the necessary vigilance to participate in the study.

For the non-suicidal clinical group, the criterion was to have at least one psychological disorder according to a structured clinical interview and the psychologist's opinion. Also, he/she should not have a history of committing suicide during the past year prior to this study. In the case of the non-clinical group, the score of less than $23 \mathrm{ob}-$ tained from a screening test, which was formulated based on the GHQ, was considered as the inclusion criterion. For all the groups, exclusion criteria were to have schizophrenia, other types of psychotic disorders, or intellectual disability. Individuals participated voluntarily in this research and they could leave it at any time. However, none of the participants discontinued participation during the research process. In addition, in order to follow ethical principles, the participants were allowed to conceal their identifying information (e.g. name and address). They could also ask to have the results if they wanted. After the groups were set, the participants completed the SST and Beck Scale of Suicide Ideation (BSS).

\section{General Health Questionnaire - 28 items (GHQ-28)}

This questionnaire is one of the most well-known screening tools in psychology that is used to identify non-psychotic disorders in various situations and different levels (Goldberg, 1972). The sensitivity and specificity of the questionnaire are reported to be $84 \%$ and $82 \%$, respectively. The reliability of this questionnaire was reported to be 0.95 (Goldberg \& Williams, 1988). In Iran, the best cut-off point of the questionnaire is 23 with a sensitivity of $88 \%$, the specificity of $79 \%$, and an overall misclassification rate of 16\% (Rezaei, Salehi, Yousefzadeh Chabok, Moosavi \& Kazemnejad, 2011). Psychometric properties of this questionnaire have been analyzed and confirmed by various international studies (Prady et al., 2013; Willmott, Boardman, Henshaw \& Jones, 2008) and studies in Iran (Rezaei et al., 2011; Noorbala, Bagheri-Yazdi \& Mohammad, 2009) using clinical as well as non-clinical samples.

\section{Beck Scale of Suicide Ideation (BSS)}

This scale is a 21-item self-report tool, based on which the intensity of suicide ideation, in adolescents and adults, can be measured (Beck \& Steer, 1991). The first 19 items are scored based on a three-point scale between zero and 2; thus, the total score of the scale is between zero and 38. Although based on the scale, the intensity of the suicide ideation and attitudes and planning towards suicide can be tested for separate groups, in most investigations, the total score is used to assess suicide ideation. The last two items assess the number of previous attempts and the seriousness of intention in the last attempt and they provide more background information for the psychiatrist; however, they are not included in the final score. The English version of the scale is highly corre- 
lated with standard depression tests and suicide attitude tests (from 0.90 for inpatients and 0.94 for outpatients).

In addition, the correlation of this scale with suicide option of depression questionnaire and Beck Hopelessness Scale and Beck Depression Questionnaire is reported to be in the range of 0.58 to 0.69 and 0.64 to 0.75 , respectively, and the Cronbach's alpha of 0.90 and 0.85 have been reported for inpatients and outpatients, respectively, indicating a high internal consistency on this scale (Beck \& Steer, 1991). In Iran, Anisi, Fathi-Ashtiani, Salimi \& Ahmadi-Noudeh (2005) assessed the simultaneous validity and reliability of the BSS in a sample of 100 individuals aged 19 to 28 years. Their findings showed that the correlation of the scale with Goldberg GHQ was 0.76 and the internal consistency, computed using Cronbach's alpha coefficient and split half-method, was 0.95 and 0.75 , respectively (Anisi et al., 2005).

\section{Emotional Stroop task (EST)- Suicide Version}

In the present research, in order to assess attentional bias, a software version of EST for suicide was built. Initially, based on the studies conducted by Cha et al. (2010) and Salehi-Fadardi \& Ziyaiee (2010), four groups of 60 words that evoke neutral emotion, positive emotion, negative emotion, and suicide were considered. The words were assessed by five psychology specialists and the amount of relevancy of the words to the corresponding emotions was scaled from 1 (not relevant at all) to 5 (completely relevant). Afterward, the words were screened based on the assigned scores, and each group of the words that had the highest score was selected and were matched in terms of the words' length, pronunciation, and the number of letters. To obtain the frequency of the words in the Persian Language, the software, created by the Institute of Humanities and Cultural Studies, was employed.

In addition, because the software was generated using older Persian texts, the frequency was also assessed using other search engines, such as Google, Yahoo, and Bing. Then, the words were given to 30 individuals who had committed suicide and were classified and scored in four groups based on the corresponding emotions. Using the scores reported by the patients, 12 words were designated for each group. Using 48 words and the Java programming language, the suicide version of the emotional stroop software was designed. On the main page of the software, two types of execution processes, including a trial test and the main test, were embedded. First, the trial test option was run for the participants and they were asked to follow the on-screen instructions. In the trial phase, initially the message "after pressing the space bar, words will appear and you should press carefully and quickly the key corresponding to their color", was shown on a grey screen.

Through pressing the space bar, first, eight words with different colors and random orders will appear on the screen to get the participant acquainted with the process. After the appearance of eight words, the message, "Test is over", will announce the end of the trial phase. The participant will be returned to the main page by pressing "OK", and then if the individual was consent, the main test was performed. For the main test, first, the message, "to start, press the space bar", will appear on the grey screen. By pressing the space bar, 48 words that were randomly arranged, based on color and meaning, will be shown and the participant should press the color key corresponding to each word. Arbitrary arrangement of the words and colors differs from one participant to another. For instance, the color of the word "glass" might be blue for one participant, while it is green for the other one.

The software is also able to save participants' reaction time to the words with an accuracy of $0.001 \mathrm{~s}$. Attentional bias is usually considered as the interference time created by the words with positive, negative, and suicidal emotions. However, in the present study, both indicators of reaction time and interference time were taken into account to score the participants' performance in the stroop test. Herein, to assess the internal consistency of the stroop test, two methods were employed. In the first method, through computing Cronbach's alpha coefficient based on reaction time data, the reliability coefficient was 0.83 for words with neutral emotion, 0.88 for words with positive emotion, 0.8 for words with negative motion, 0.89 for words related to suicide, and 0.946 for all the words. Afterward, the method of split-half reliability with Spearman-Brown Correction and classification of the items into two groups of odd and even was employed. The reliability coefficient obtained from this method was 0.68 for words with neutral emotion, 0.89 for words with positive emotion, 0.85 for words with negative emotion, 0.92 for words related to suicide, and 0.94 for all the words.

The data, collected using descriptive statistical methods, are summarized were presented in form of frequency values (f), frequency percentage (\%), Mean $\pm \mathrm{SD}$, tables, and figures. In the analysis, first, the data distribution in each group was determined using the ShapiroWilk test. Then, using default values for the parametric tests, Multivariate Variance Analysis (MANOVA) was performed to assess the difference between the groups in terms of the research variables. Also, the analysis of 
simultaneous discriminant function was used to analyze the role of attentional bias in predicting suicide. It should be noted that all the analysis was performed using SPSS V. 22 at $95 \%$ confidence level.

\section{Results}

Table 2 presents descriptive statistics associated with the research variables for suicide clinical group, nonsuicide clinical group, non-clinical group, and the whole sample. According to Table 2, the interference time was negative for positive and negative emotional stroop; that is, in each group, the reaction time to positive and negative emotional words was shorter than that to neutral words and there was no delay in the effect of positive and negative emotional words. The fastest and the slowest reaction time, for the positive stroop, was respectively associated with the non-suicidal clinical group (Mean \pm SD: $-59.302 \pm 219.64$ ) and suicidal clinical group (Mean \pm SD: $-5.708 \pm 123.22$ ) and for negative stroop, it corresponded respectively to the non-suicidal clinical group (Mean \pm SD:-15.977 \pm 196.69 ) and non-clinical group (Mean \pm SD:-0.143 \pm 70.25 ).

Table 1. Characteristic of the study participants (n:120)

\begin{tabular}{|c|c|c|c|c|c|}
\hline \multicolumn{6}{|c|}{ No. (\%) } \\
\hline \multicolumn{2}{|c|}{ Variables } & \multirow{2}{*}{$\begin{array}{c}\begin{array}{c}\text { Clinical Suicidal } \\
\text { (n:40) }\end{array} \\
14(35.0)\end{array}$} & \multirow{2}{*}{$\begin{array}{c}\begin{array}{c}\text { Non Clinical } \\
\text { Suicidal (n:40) }\end{array} \\
12(30.0)\end{array}$} & \multirow{2}{*}{$\begin{array}{c}\begin{array}{c}\text { Non Clinical } \\
\text { (n:40) }\end{array} \\
18(45.0)\end{array}$} & \multirow{2}{*}{$\begin{array}{c}\begin{array}{c}\text { Total Sample } \\
\text { (n:120) }\end{array} \\
44(36.7)\end{array}$} \\
\hline & $<20$ & & & & \\
\hline \multirow[t]{2}{*}{ Age } & $20-30$ & $23(57.5)$ & $20(50.0)$ & $20(50.0)$ & $63(52.5)$ \\
\hline & $>30$ & $3(7.5)$ & $8(20.0)$ & $2(5.0)$ & $13(10.8)$ \\
\hline \multirow{2}{*}{ Gender } & Female & $20(50.0)$ & $30(75.0)$ & $27(67.5)$ & $77(64.2)$ \\
\hline & Male & $20(50.0)$ & $10(25.0)$ & $13(32.5)$ & $43(35.8)$ \\
\hline \multirow{4}{*}{ Marital Status } & Single & $25(62.5)$ & $28(70.0)$ & $30(75.0)$ & $83(69.2)$ \\
\hline & Married & $13(32.5)$ & $9(22.5)$ & $10(25.0)$ & $32(26.7)$ \\
\hline & $\begin{array}{l}\text { Divorced/ Wid- } \\
\text { owed }\end{array}$ & $1(2.5)$ & $3(7.5)$ & - & $4(3.3)$ \\
\hline & Remarried & $1(2.5)$ & - & - & $1(0.8)$ \\
\hline \multirow{7}{*}{ Education } & Primary school & $4(10.0)$ & - & - & $4(3.3)$ \\
\hline & Middle school & $8(20.0)$ & $6(15.0)$ & - & $14(11.7)$ \\
\hline & High school & $7(17.5)$ & $5(12.5)$ & $14(35.0)$ & $26(21.7)$ \\
\hline & diploma & $13(32.5)$ & $10(25.0)$ & $18(45.0)$ & $41(34.2)$ \\
\hline & Associate degree & $4(10.0)$ & $2(5.0)$ & $3(7.5)$ & $9(7.5)$ \\
\hline & University degree & $3(7.5)$ & $16(40.0)$ & $4(10.0)$ & $23(19.2)$ \\
\hline & Master's degree & $1(2.5)$ & $1(2.5)$ & $1(2.5)$ & $3(2.5)$ \\
\hline \multirow{5}{*}{ Occupation } & Unemployed & $5(12.5)$ & $4(10.0)$ & $1(2.5)$ & $10(8.3)$ \\
\hline & Employed & $16(40.0)$ & $12(30.0)$ & $10(25.0)$ & $38(31.7)$ \\
\hline & Student & $13(32.5)$ & $13(32.5)$ & $27(67.5)$ & $53(44.2)$ \\
\hline & Housewife & $6(15.0)$ & $10(25.0)$ & $2(5.0)$ & $18(15.0)$ \\
\hline & Disabled & - & $1(2.5)$ & - & $1(0.8)$ \\
\hline
\end{tabular}


Different trends were observed in the values of suicide stroop. For suicidal clinical and non-clinical groups, the reaction time was longer for suicide-related words in comparison with neutral emotional words; however, a similar trend was not observed in the non-suicidal clinical group. As expected, the longest reaction time and consequently, the longest interference time in case of suiciderelated words, happened for the group of clinical suicide (Mean \pm SD: $32.887 \pm 130.76$ ). Regardless of the types of the groups, the fastest reaction time was related to positive emotional stroop (Mean \pm SD: $-24.325 \pm 151.12$ ) and the slowest reaction time was associated with suicide stroop (Mean \pm SD: $11.58 \pm 160.30$ ). Mean \pm SD values of reaction time, interference time, and group interference ratio are displayed in Table 1 for different types of stroop.

Afterward, the MANOVA was performed to compare the difference between the groups in terms of mean reaction time level, mean interference level for different types of stroop, and suicidal thinking level. Because the reaction speed and the interference level can be highly correlated, the MANOVA was conducted in two steps: once with interference time variables and once with reaction time variables. The summary of the results is presented in the following tables. Initially, the defaults, required to do the analysis, were assessed and the results are shown in Table 3. Based on the table and the results obtained from Levene's test, Box's M test (Mean: 251.755, $\mathrm{P}<0.000$ ), and Bartlett's test (K2 3819.097; $\mathrm{P}<0.000)$, required assumptions were met to perform the test.

The results of the MANOVA are presented in Table 4. For the variables associated with the reaction time of 0.979 with the indicator factor $(\mathrm{F})$ of 1067.243 and 5 and 113 degrees of freedom, it can be seen that Pillai's trace effect size was statistically significant $(\mathrm{P}<0.000)$ and 0.979 of the total variance was explained by the independent variable. Moreover, for the variables associated with interference time of 0.979 with the indicator factor $(F)$ of 438.607 and 4 and 114 degrees of freedom, it can be seen that Java programming language trace effect size was statistically meaningful $(\mathrm{P}<0.000)$ and it can be inferred that 0.939 of the total variance was explained by the independent variable. Hence, the multivariate test was statistically meaningful and each dependent variable was separately evaluated.

The results obtained from the intergroup effect test, which separately measured each of the dependent variables, are presented in Table 5. According to this table, among dependent variables, interference time variable in suicide stroop with $\mathrm{F}=0.569$ and $\mathrm{P}<0.568$, in positive stroop with $\mathrm{F}=1.626$ and $\mathrm{P}<0.201$, and in negative stroop with $\mathrm{F}=0.147$ and $\mathrm{P}<0.863$ was not statistically significant among the three groups. However, for all the groups, the reaction time variables in suicide stroop with $\mathrm{F}=12.759$ and $\mathrm{P}<0.000$, in positive stroop with $\mathrm{F}=18.520$

Table 2. Mean $\pm S D$ of the Variables

\begin{tabular}{|c|c|c|c|c|}
\hline \multirow{2}{*}{ Variables } & \multicolumn{4}{|c|}{ Mean $\pm S D$} \\
\hline & Suicidal Clinical & Non-suicidal Clinical & Non-clinical & Total Sample \\
\hline Age & $22.53 \pm 7.23$ & $25.85 \pm 7.76$ & $22.65 \pm 6.57$ & $23.67 \pm 7.31$ \\
\hline Years of education & $11 \pm 2.68$ & $13.37 \pm 2.61$ & $12.95 \pm 2.02$ & $12.44 \pm 2.65$ \\
\hline GHQ-28 & - & - & $13.63 \pm 5.81$ & - \\
\hline Suicide ideation & $15.55 \pm 9.39$ & $5.90 \pm 6.83$ & $3.29 \pm 2.35$ & $7.93 \pm 8.89$ \\
\hline Reaction time in neutral stroop & $1070.57 \pm 220.06$ & $943.01 \pm 307.69$ & $863.75 \pm 120.61$ & $959.11 \pm 242.87$ \\
\hline Reaction time in positive stroop & $1064.86 \pm 208.60$ & $883.71 \pm 164.09$ & $855.78 \pm 114.22$ & $934.79 \pm 189.80$ \\
\hline Reaction time in negative stroop & $1063.34 \pm 234.08$ & $927.03 \pm 214.93$ & $863.61 \pm 112.63$ & $951.33 \pm 210.37$ \\
\hline Reaction time in suicide stroop & $1103.46 \pm 266.66$ & $938.67 \pm 238.17$ & $869.97 \pm 97.60$ & $970.70 \pm 233.87$ \\
\hline Interference time in positive stroop & $-5.708 \pm 123.22$ & $-59.302 \pm 219.64$ & $-7.966 \pm 66.13$ & $-24.325 \pm 151.12$ \\
\hline $\begin{array}{l}\text { Interference time in negative } \\
\text { stroop }\end{array}$ & $-7.231 \pm 87.93$ & $-15.997 \pm 196.69$ & $-0.143 \pm 70.25$ & $-7.784 \pm 129.89$ \\
\hline Interference time in suicide stroop & $32.887 \pm 130.76$ & $-4.337 \pm 230.08$ & $6.21 \pm 87.30$ & $11.58 \pm 160.30$ \\
\hline
\end{tabular}


Table 3. Results of Levene's, Box's M and Bartlett's Tests

\begin{tabular}{|c|c|c|c|c|c|c|c|}
\hline Variable & $\mathbf{F}$ & Df1 & Df2 & Sig. & M Box & $\begin{array}{l}\text { Likelihood } \\
\text { Ratio }\end{array}$ & $\begin{array}{c}\text { Approx. } \\
\text { Chi-square }\end{array}$ \\
\hline Suicide ideation & 27.352 & 2 & 117 & 0.000 & - & - & - \\
\hline Interference time in suicide stroop & 1.767 & 2 & 117 & 0.175 & - & - & - \\
\hline Interference time in positive stroop & 2.039 & 2 & 117 & 0.135 & - & - & - \\
\hline Interference time in negative stroop & 1.739 & 2 & 117 & 0.180 & - & - & - \\
\hline Reaction time in suicide stroop & 13.529 & 2 & 117 & 0.000 & - & - & - \\
\hline Reaction time in positive stroop & 7.596 & 2 & 117 & 0.001 & - & - & - \\
\hline Reaction time in negative stroop & 7.217 & 2 & 117 & 0.001 & - & - & - \\
\hline Reaction time in neutral stroop & 3.341 & 2 & 117 & 0.039 & - & - & - \\
\hline Box's M test & 3.777 & 56 & 39100.728 & & 231.306 & - & - \\
\hline Bartlett's test & - & 27 & - & 0.000 & - & 0.000 & 3050.732 \\
\hline
\end{tabular}

and $\mathrm{P}<0.000$, in negative stroop with $\mathrm{F}=10.955$ and $\mathrm{P}<0.000$ and in neutral stroop with $\mathrm{F}=8.288$ and $\mathrm{P}<0.000$ showed a significant difference. Suicide ideation variable with $\mathrm{F}=38.374$ and $\mathrm{P}<0.000$ had a significant difference for all the groups.

Discriminant function analysis was employed to assess the possibility to predict the three groups based on reaction interference time and reaction time speed. According to the nature of the discriminant function and also due to non-meaningful values for reaction interference time, obtained from MANOVA, these variables were excluded from discriminant analysis and the process proceeded using variables of reaction time speed and suicide ideation. The summary of the results associated with discriminant function, using simultaneous analysis (three variables), are reported in Table 6.

According to the data presented in Table 6 and the groups' characteristics, two discriminant functions were created. The eigenvalue of the first function, which distinguishes the suicidal clinical group, non-suicidal clinical group, and non-clinical group, was 0.966 with a canonical correlation of 0.701 and determined $95.7 \%$ of the variance. The Chi-square value was 82.635 , which was significant at the level of 0.001 . Therefore, the first func-

Table 4. Multivariate tests

\begin{tabular}{|c|c|c|c|c|c|c|c|c|}
\hline & Variable & & Value & $\mathbf{F}$ & Df & Error df & Sig. & $\begin{array}{l}\text { Partial Eta } \\
\text { Squared }\end{array}$ \\
\hline \multirow{5}{*}{ Reaction time } & Intercept & $\begin{array}{l}\text { Java programming } \\
\text { language Trace }\end{array}$ & 0.979 & 1067.243 & 5 & 113 & 0.000 & 0.979 \\
\hline & & Wilks' Lambda & 0.021 & 1067.243 & 5 & 113 & 0.000 & 0.979 \\
\hline & & & & & & & & \\
\hline & Groun & Pillai's Trace & 0.533 & 8.283 & 10 & 228 & 0.000 & 0.266 \\
\hline & & Wilks' Lambda & 0.444 & 7.861 & 10 & 226 & 0.000 & 0.302 \\
\hline \multirow{5}{*}{ Interference time } & Intercept & Pillai's Trace & 0.939 & 438.607 & 4 & 114 & 0.000 & 0.939 \\
\hline & & Wilks' Lambda & 0.061 & 438.607 & 4 & 114 & 0.000 & 0.939 \\
\hline & & Pillai's Trace & 0.443 & 8.189 & 8 & 230 & 0.000 & 0.222 \\
\hline & Group & & & & & & & \\
\hline & & Wilks' Lambda & 0.572 & 9.192 & 8 & 228 & 0.000 & 0.244 \\
\hline
\end{tabular}


Table 5. Tests of between-subjects effects of research variables

\begin{tabular}{|c|c|c|c|c|c|c|}
\hline Dependent Variable & Type III Sum of Squares & Mean Square & Df & $\mathbf{F}$ & Sig. & $\begin{array}{c}\text { Partial Eta } \\
\text { Squared }\end{array}$ \\
\hline Suicide ideation & 3732.867 & 1866.433 & 2 & 38.374 & 0.000 & 0.396 \\
\hline Interference time in suicide stroop & 29445.659 & 14722.829 & 2 & 0.569 & 0.568 & 0.010 \\
\hline Interference time in positive stroop & 73502.686 & 36751.434 & 2 & 1.626 & 0.201 & 0.027 \\
\hline Interference time in negative stroop & 5032.223 & 2516.111 & 2 & 0.147 & 0.863 & 0.003 \\
\hline Reaction time in suicide stroop & 1151908.543 & 575954.272 & 2 & 12.759 & 0.000 & 0.177 \\
\hline Reaction time in positive stroop & 1030809.809 & 515404.904 & 2 & 18.520 & 0.000 & 0.240 \\
\hline Reaction time in negative stroop & 833277.242 & 416638.621 & 2 & 10.955 & 0.000 & 0.158 \\
\hline Reaction time in neutral stroop & 871049.742 & 435523.871 & 2 & 8.288 & 0.000 & 0.124 \\
\hline
\end{tabular}

Table 6. Summary of Canonical Discriminant Functions: Eigenvalues and Wilk's Lambda

\begin{tabular}{cccccccc}
\hline Function & Eigenvalue & Variance (\%) & Cumulative (\%) & Canonical Correlation & Wilks' Lambda & Chi-square & Df \\
\hline 1 & 0.966 & 95.7 & 95.7 & 0.701 & 0.487 & 82.635 & 10 \\
2 & 0.043 & 4.3 & 100 & 0.204 & 0.959 & 4.873 & 4 \\
\hline & & & & & $\begin{array}{l}\text { PRACTICE In } \\
\text { CLINICAL PSYCH LOGY }\end{array}$ \\
\hline
\end{tabular}

tion distinguished properly the suicidal clinical group, non-suicidal clinical group, and non-clinical group. The second function had the eigenvalue of 0.043 and its canonical correlation was 0.204 and it determined $4.3 \%$ of the variance. The Chi-square value for this function was 4.873, which was significant at the level of 0.001 . Thus, the second function makes a weak difference between the first function and the non-clinical group.

Table 7 displays standardized, structure, and unstandardized coefficients of entering discriminant function for the three sample groups. Using structure coefficients, the prediction power of each predictive variable for the discriminant function can be observed. According to this table, variables of suicide ideation (0.470), reaction time in positive stroop (0.571), reaction time in suicide stroop $(0.470)$, reaction time in negative stroop (0.438), and reaction time in neural stroop (0.376) were involved in distinguishing between suicide clinical group and nonsuicidal clinical, and non-clinical groups, respectively.

Table 8 shows that the discriminant function correctly predicted the membership, in $67.5 \%$ of cases for suicidal clinical individuals, in $42.5 \%$ of cases for non-suicidal clinical individuals, and in $75 \%$ of cases for non-clinical individuals. Overall, 74 cases out of 120 patients were correctly categorized. In other words, the success rate of the discriminant function was equal to $61.7 \%$.

\section{Discussion}

The main objective of the present research was to assess and compare the predictive role of attentional bias in suicidal clinical, suicidal non-clinical, and non-clinical patients. The results indicated that analysis of attentional bias, based on the interference effect, does not provide a significant difference in the three groups; thus, it cannot play a significant role in predicting the groups. However, when reaction time is used as the criterion, all the three groups of words with emotional stroop (suicide, positive, and negative) caused a significant difference between the groups. In other words, the meaning of the words draws individuals' attention towards itself. All three groups of the words also had the ability to predict and distinguish between the groups.

However, the group of positive words had a prominent role compared with the group of suicide words. These results are not in accordance with some of the previous investigations, such as those conducted by Williams \& Bordbent (1986), Becker et al. (1999), Cha et al. (2010), Steward et al. (2017), and Wilson et al. (2019) who re- 
Table 7. Standardized canonical discriminant function coefficients, structure matrix, and canonical discriminant functions coefficients

\begin{tabular}{|c|c|c|c|}
\hline & \multirow{2}{*}{ Variables } & \multicolumn{2}{|c|}{ Functions } \\
\hline & & 1 & 2 \\
\hline \multirow{5}{*}{ Standardized coefficients } & Suicide ideation & 0.813 & 0.246 \\
\hline & Reaction time in suicide stroop & 0.102 & 0.694 \\
\hline & Reaction time in positive stroop & 0.721 & -1.902 \\
\hline & Reaction time in negative stroop & -0.241 & 0.638 \\
\hline & Reaction time in neutral stroop & -0.059 & 0.740 \\
\hline \multirow{5}{*}{ Structure matrix } & Suicide ideation & 0.822 & 0.251 \\
\hline & Reaction time in suicide stroop & 0.470 & 0.205 \\
\hline & Reaction time in positive stroop & 0.571 & -0.203 \\
\hline & Reaction time in negative stroop & 0.438 & 0.245 \\
\hline & Reaction time in neutral stroop & 0.376 & 0.244 \\
\hline \multirow{7}{*}{ Canonical coefficients } & Suicide ideation & 0.117 & 0.035 \\
\hline & Reaction time in suicide stroop & 0.000 & 0.003 \\
\hline & Reaction time in positive stroop & 0.004 & -0.011 \\
\hline & & & \\
\hline & Reaction time in negative stroop & -0.001 & 0.003 \\
\hline & Reaction time in neutral stroop & -0.000 & -0.003 \\
\hline & (Constant) & -6.212 & 0.346 \\
\hline
\end{tabular}

ported that attentional bias in suicide stroop is related to a suicide attempt. However, these results are in accordance with studies by Chung \& Jeglic (2016), RichardDiwanto et al. (2016), and Chung and Jeglic (2016).

The inconsistencies can be mainly attributed to the differences, associated with the characteristics of studied samples and the scoring methods. For example, Williams \& Broadbent (1986), through comparing attentional bias, based on delay reaction time in Autobiographical Memory Retrieval Test between the suicidal clinical group, non-suicidal clinical group, and non-clinical group, found out that those with a history of suicide suffer from retrieval bias in the reaction to positive stimulus words in comparison with other two groups. However, this bias was only observed for the non-clinical group with respect to negative stimulus words. In addition, Becker et al. (1999) compared attentional bias in neutral stroop, positive emotional stroop, negative emotional stroop, and suicide stroop between the suicidal clinical group and non-clinical group. Our results showed that the reaction time was longer in case of the clinical group in suicide stroop in comparison with other types of stroop, which clearly indicates an attentional bias towards the suicide-related information for the clinical group, while for the non-clinical group, there was no difference in reaction time to different types of stroop.

On the other hand, Chung and Jeglic (2016) found that the interference time, in negative emotional stroop and suicide stroop, does not have a significant correlation with suicide ideation and the bias, towards suicide words, is only correlated with suicide ideation for females $(\mathrm{r}=0.12)$. Moreover, Richard-Divantoy et al. (2016) did not observe a meaningful difference in interference time in suicide stroop between two groups of with and without suicide ideation; in other words, there was not a significant relation between interference time and suicide ideation. Similar to these observations, Chung and Jeglic (2016), Richard-Divantoy et al. (2016), and Wilson et 
Table 8. Classification results of discriminant function

\begin{tabular}{cccc}
\hline \multirow{2}{*}{ Groups } & \multicolumn{3}{c}{ No. (\%) } \\
\cline { 2 - 4 } & Suicidal Clinical & Non-suicidal Clinical & Non-clinical \\
\hline Suicidal clinical & $27(67.5)$ & $8(20)$ & $5(12.5)$ \\
Non-suicidal clinical & $6(15)$ & $17(42)$ & $17(43.5)$ \\
Non-clinical & $0(0)$ & $10(25)$ & 30 (75) \\
\hline
\end{tabular}

al. (2019) did not report a significant difference between reaction time in neutral, positive emotional stroop, negative emotional stroop, and suicide stroop, which was not in accordance with the findings of the present research. Recently, inconsistencies in research findings have raised the question of whether the SST, in terms of psychometric properties (particularly reliability) and the type of scoring method, which are reported for suicide stroop, has played a role in the occurrence of these discrepancies.

In these investigations, using suicide stroop, some other indicators were employed to score the participants in suicide stroop. For instance, they used raw reaction time (Becker et al., 1999), interference time compared with neutral stroop, through which reaction time difference for various types of stroop is computed using neutral reaction time (Cha et al., 2010; Cha et al., 2017; Cha et al., 2010, 2017), and the ratio of interference time to reaction time in neutral stroop (Stewart et al., 2017). Accordingly, in a comprehensive investigation, Wilson et al. (2019) reviewed the psychometric properties of SST along with other analyses, based on the data obtained from the previous seven studies. The results of assessing the reliability of SST using the split-half reliability method revealed that the reliability coefficients of various types of stroop is between 0.93 and 0.94 for the whole sample and the sub-groups (clinical/non-clinical, adults/youth) and based on the reaction time scoring method;

Reliability coefficient is very low based on different scoring methods (i.e. computing interference time and interference ratio). In terms of concurrent validity, researchers compared attentional bias towards suicide stroop based on different scoring systems and in two separate series of analyses for two types of categories (suicidal/non-suicidal and suicidal/suicide ideation/control). According to the results obtained from the analyses, the interactive effect of the group and stroop type on the attentional bias is not statistically significant based on none of the scoring methods for the two categories. However, based on the reaction time scoring method, the effect of the group is significant on attentional bias in both dual and triple categories.

Testing time is another justification to prove that attentional bias only sometimes predicts suicide attempts. In these studies, attentional bias was usually assessed after a suicide attempt using corresponding tools. Assessing attentional bias after the attempt- when for many people, the crisis is resolved- is probably very different from the bias, experienced at the time that the person is really suicidal. Many of those people, who committed suicide, are happy to be alive after a failed attempt. These observations suggest that patients' feelings, regarding their suicide attempt, might distort the results and influence their bias.

The tool, used to assess attentional bias, can also be another reason for the inconsistencies in the results of attentional bias based on emotional stroop. As Harvey, Watkins, Mansell and Shafran (2004) emphasize, the task of sign searching measures attentional bias and selective attention, more properly than emotional stroop. It should be noted that in the present research, the task of emotional stroop was presented to the individuals using a laptop. It is possible that the results (where reaction speed was measured with the accuracy of $0.001 \mathrm{~s}$ ) were affected either by the lack of sufficient expertise and skills in working with computers or because this task was presented in public (hospital, office, and classroom) to the individuals. Also, the reaction time could be increased due to some distracting stimuli; thus, it was clear that the difference between the groups would not be bold.

Another variable that affected the results was the ability or defect in visual perception, visual skills, and visual information retrieval strategies that can affect a person's performance on the stroop test. In this study, this issue was not under the control of researchers.

Also, taking or the lack of using the drug at the time of the stroop test and also having comorbid mental disorders with suicide attempts can affect the results. 
In summary, it can be concluded that due to the limited number of investigations, the available knowledge has substantial limitations, and based on the available information, the relationship between attentional bias and suicide and the suicide attempt is not a result of a particular pattern. The reason can be attributed to the role of mediating variables (interfering variables related to the test site, variables related to the subjects themselves, such as visual skills deficits, taking drugs that can affect the speed of cognitive processing, having different cultural backgrounds, and having personality disorders or other mental disorders that affect test results), which needs further investigations. On the other hand, suicide is a phenomenon, which despite its different types (impulsive and based on ideation), is resulted from many implicit and explicit variables. The type of suicide attempt can influence cognitive processes and also change attentional bias.

According to the findings of the present research, it seems that using reaction time indicator can provide better psychometric properties as well as higher analyzing power. However, for future studies, psychometric properties as well as the predictive power of SST should be assessed more extensively using all three scoring methods (reaction time, interference time, and interference ratio).

\section{Conclusion}

In the present research, clinical and non-clinical populations, studied in suicide stroop concerning interference, showed a significant difference; however, they were different in terms of reaction time. Hence, it seems that the assessment of attentional bias, using emotional stroop concerning suicide-related topics, is not a function of the clinical situation and other factors, such as scoring method, are involved and further extensive investigations need to be conducted in this area.

\section{Ethical Considerations}

\section{Compliance with ethical guidelines}

The patients completed the ethical guideline form.

\section{Funding}

This research did not receive any grant from funding agencies in the public, commercial, or non-profit sectors.

\section{Authors' contributions}

All authors contributed in preparing this article.

\section{Conflict of interest}

The authors declared no conflict of interest.

\section{Acknowledgments}

We would like to express our sincere gratitude to the staff of Hospitals, and Medical Centers in Tehran for their collaboration in this research.

\section{References}

Anisi, J., Fathi-Ashtiani, A., Salimi, Sh., \& Ahmadi-Noudeh , Kh. (2005). Validity and reliability of Beck Suicide Scale Ideation among soldiers. Journal of Military Medicine, 7(1), 33-7. https:// www.sid.ir/fa/journal/ViewPaper.aspx?id=22790

Beck, A. T., Kovacs, M., \& Weissman, A. (1979). Assessment of suicidal intention: The scale for suicide ideation. Journal of Consulting and Clinical Psychology, 47(2), 343-52. [DOI:10.1037/0022006X.47.2.343] [PMID]

Balazs, J., \& Kereszteny, A. (2017). Attention-deficit/hyperactivity disorder and suicide: A systematic review. World Journal of Psychiatry, 7(1), 44-59. [DOI:10.5498/wjp.v7.i1.44] [PMID] [PMCID]

Beck, A. T., \& Steer, R. A. (1991). Manual for the beck scale for suicide ideation. San Antonio: Psychological Corporation. https://books. google.com/books?id=bFFSHAAACAAJ\&dq

Becker, E. S., Strohbach, D., \& Rinck, M. (1999). A specific attentional bias in suicide attempters. The Journal of Nervous and Mental Disease, 187(12), 730-5. [DOI:10.1097/00005053-199912000-00004] [PMID]

Cha, C. B., Najmi, S., Park, J. M., Finn, C. T., \& Nock, M. K. (2010) Attentional bias toward suicide-related stimuli predicts suicidal behavior. Journal of Abnormal Psychology, 119(3), 616-22. [DOI:10.1037/a0019710] [PMID] [PMCID]

Cha, C. B., Naimi, S., Amir, N., Matthews, J. D., Deming, C. A., Glenn J. J., \& et al. (2017). Testing the efficacy of attention bias modification for suicidal thoughts: Findings from two experiments. $A r-$ chives of Suicide Research, 21(1), 33-51. [DOI:10.1080/13811118.2016 .1162241] [PMID]

Chung, Y., \& Jeglic, E. L. (2016). Use of the modified emotional stroop task to detect suicidality in college population. Suicide and Life-Threatening Behavior, 46(1), 55-66. [DOI:10.1111/sltb.12174] [PMID]

Cisler, J. M., Bacon, A. K., \& Williams, N. L. (2009). Phenomenological characteristics of attentional biases towards threat: A critical review. Cognitive Therapy and Research, 33(2), 221-34. [DOI:10.1007/ s10608-007-9161-y] [PMID] [PMCID]

Cohen, J. (1988). Statistical power analysis for the behavioral sciences. $2^{\text {nd }}$ Ed. Hillsdale, NJ: Lawrence Erlbaum Associates. https://books. google.com/books?id=cIJH01R33bgC\&dq

Cyders, M. A., \& Coskunpinar, A. (2011). Measurement of constructs using self-report and behavioral lab tasks: Is there overlap in nomothetic span and construct representation for impulsivity? Clinical Psychology Review, 31(6), 965-82. [DOI:10.1016/j. cpr.2011.06.001] [PMID] 
Davidson, J. R. T. (2000). Social anxiety disorder under scrutiny. Depression and Anxiety, 11(3), 93-8. [DOI:10.1002/(SICI)15206394(2000)11:3<93::AID-DA2>3.0.CO;2-7] [PMID]

DeVito, E. E., Kiluk, B. D., Nich, C., Mouratidis, M., \& Carroll, K. M. (2018). Drug Stroop: Mechanisms of response to computerized cognitive behavioral therapy for cocaine dependence in a randomized clinical trial. Drug and Alcohol Dependence, 183, 162-8. [DOI:10.1016/j.drugalcdep.2017.10.022] [PMID] [PMCID]

Drobes, D. J., Oliver, J. A., Correa, J. B., \& Evans, D. E. (2019). Attentional bias and smoking. In V. R. Preedy (Ed.), Neuroscience of nicotine: Mechanisms and treatment (p. 145-150). Cambridge, MA: Academic Press. [DOI:10.1016/B978-0-12813035-3.00018-6]

Familoni, B. O., Ma, L., Andrew Hutchinson, J., Andrew Morgan III, C., Rasmusson, A., \& O'Kane, B. L. (2012). SAFE for PTSD: Noncontact psychophysiological measure based on high-resolution thermal imaging to aid in PTSD diagnosis and assessment of treatment. Paper presented at SPIE 8401, Independent Component Analyses, Compressive Sampling, Wavelets, Neural Net, Biosystems, and Nanoengineering X, 840115, 10 May 2012, Baltimore, Maryland, United States. [DOI:10.1117/12.926464]

Goldberg, D. P. (1972). The detection of psychiatric illness by questionnaire: A technique for the identification and assessment of nonpsychotic psychiatric illness. London: Oxford University Press. https:/ / books.google.com/books?id=PbRrAAAAMAAJ\&q

Goldberg, D. P., \& Hillier, V. F. (1979). A scaled version of the general health questionnaire. Psychological Medicine, 9(1), 139-45. [DOI:10.1017/S0033291700021644] [PMID]

Goldberg, D., \& Williams, P. (1988). A user's guide to the General Health Questionnaire. Windsor: NFER.

Goodman, M., \& Hazlett, E. High risk suicidal behavior in veterans-assessment of predictors and efficacy of dialectical behavioral therapy. New York: VA Medical Center Bronx United States; 2015.

Hamedi, A., Colborn, V. A., Bell, M., Chalker, S. A., \& Jobes, D. A. (2019). Attentional bias and the Suicide Status Form: Behavioral perseveration of written responses. Behaviour Research and Therapy, 120, 103403. [DOI:10.1016/j.brat.2019.04.011] [PMID]

Harvey, A., Watkins, E., Mansell, W., \& Shafran, R. (2004). Cognitive behavioral processes across psychological disorders: A transdiagnostic approach to research and treatment. New York: Oxford University Press. [DOI:10.1093/med:psy ch/9780198528883.001.0001]

Nock, M. K., Park, J. M., Finn, C. T., Deliberto, T. L., Dour, H. J., \& Banaji, M. R. (2010). Measuring the suicidal mind: Implicit cognition predicts suicidal behavior. Psychological Science, 21(4), 511-7. [DOI:10.1177/0956797610364762] [PMID] [PMCID]

Noorbala, A. A., Bagheri-Yazdi, S. A., \& Mohammad, K. (2009). [The validation of general health questionnaire-28 as a psychiatric screening tool (Persian)]. Hakim Research Journal, 11(4), 47-53. https://www.sid.ir/fa/journal/ViewPaper. aspx?ID $=89228$

Prady, S. L., Miles, J. N. V., Pickett, K. E., Fairley, L., Bloor, K., Gilbody, S., et al. (2013). The psychometric properties of the subscales of the GHQ-28 in a multi-ethnic maternal sample: Results from the Born in Bradford cohort. BMC Psychiatry, 13, 55. [DOI:10.1186/1471-244X-13-55] [PMID] [PMCID]
Rezaei, S., Salehi, I., Yousefzadeh Chabok, Sh., Moosavi, H., \& Kazemnejad, E. (2011). [Factor structure, clinical cut off point and psychometric properties of 28 -items version for general health questionnaire in patients with traumatic brain injury (Persian)]. Journal of Guilan University of Medical Sciences, 20(78), 56-70. http://journal.gums.ac.ir/article-1-148-en.htm

Richard-Devantoy, S., Ding, Y., Turecki, G., \& Jollant, F. (2016). Attentional bias toward suicide-relevant information in suicide attempters: A cross-sectional study and a meta-analysis. Journal of Affective Disorders, 196, 101-8. [DOI:10.1016/j. jad.2016.02.046] [PMID]

Salehi-Fadardi, J., \& Ziyaie, S. S. (2010). [Implicit cognitive processes and attention bias toward addictive behaviors: Introduction, development and application of addiction stroop test (Persian)]. Journal of Fundamentals of Mental Health, 12(45), 35889. [DOI:10.22038/JFMH.2010.886]

Stewart, J. G., Glenn, C. R., Esposito, E. C., Cha, C. B., Nock, M K., \& Auerbach, R. P. (2017). Cognitive control deficits differentiate adolescent suicide ideators from attempters. The Journal of Clinical Psychiatry, 78(6), e614-21. [DOI:10.4088/ JCP.16m10647] [PMID]

Turner, S. M., \& Beidel, D. C. (2004). Treating obsessive-compulsive disorder. New York: Pergamon Press, Inc.

Wenzel, A., Brown, G. K., \& Beck, A. T. (2009). Cognitive therapy for suicidal patients: Scientific and clinical applications. Washington, DC: American Psychological Association. [DOI:10.1037/11862-000]

Williams, J. M., \& Broadbent, K. (1986). Autobiographical memory in suicide attempters. Journal of Abnormal Psychology, 95(2), 144-9. [DOI:10.1037/0021-843X.95.2.144] [PMID]

Willmott, S., Boardman, J., Henshaw, C., \& Jones, P. (2008). The predictive power and psychometric properties of the General Health Questionnaire (GHQ-28). Journal of Mental Health, 17(4), 435-42. [DOI:10.1080/09638230701528485]

Wilson, K. M., Millner, A. J., Auerbach, R. P., Glenn, C. R., Kearns, J. C., Kirtley, O. J., \& et al. (2019). Investigating the psychometric properties of the Suicide Stroop Task. Psychological Assessment, 31(8), 1052-61. [DOI:10.1037/pas0000723] [PMID] [PMCID]

World Health Organization. (2019). Suicide data. Retrieved from https://www.who.int/mental_health/prevention/suicide/suicideprevent/en

Young Journalists Club. (2018). [Suicide statistics in Iran (Persian)]. Retrieved from https://www.yjc.ir/fa/ news/6660232/ 
This Page Intentionally Left Blank 